\title{
Hemorrhagic pseudoaneurysm in a pancreatic pseudocyst after extracorporeal shock wave lithotripsy for pancreatolithiasis
}

A 49-year-old man with a history of alcohol abuse and chronic pancreatitis was referred to our hospital for extracorporeal shock wave lithotripsy (ESWL) for pancreatolithiasis. Computed tomography (CT) revealed chronic pancreatitis with a pseudocyst (diameter, $30 \mathrm{~mm}$ ) in the pancreatic tail and multiple pancreatic calculi (ه Fig. 1 a,b).

We initially performed an endoscopic pancreatic sphincterotomy then administered four courses of ESWL without noting any remarkable improvement in the pancreatolithiasis.

The patient experienced sudden, severe upper abdominal pain 6 days after the fourth course of ESWL. A contrast-enhanced CT scan showed enlargement of the pseudocyst (diameter, $50 \mathrm{~mm}$ ) and a hemorrhagic pseudoaneurysm in the cyst ( $\bullet$ Fig. 1 c, d). Selective celiac angiography revealed a pseudoaneurysm in the pancreatic branch of the splenic artery ( $\bullet$ Fig. 2 ). Transcatheter arterial embolization with a mixture of n-butyl-2-cyanoacrylate and iodized oil resulted in hemostasis of the pseudoaneurysm. During a 3-year followup, he experienced no further bleeding.

Hemorrhagic pseudoaneurysm in a pancreatic pseudocyst is a serious complication of pancreatitis, with a reported mortality rate of $18 \%-29 \%$ in treated patients and $>90 \%$ in untreated patients [1]. Pseudoaneurysms are formed by the enzymerich peripancreatic fluid of the pseudocyst, which causes autodigestion and weakening of the walls of the adjacent arteries [2]. Although ESWL is a highly effective and safe treatment for calculi of the main pancreatic duct [3] and other organs, renal hematoma is one of the most significant adverse effects of ESWL [4]. This renal hemorrhage suggests that ESWL causes renal cellular injury and ultrastructural damage in kidney tissues [4]. In our case, the appearance of the pseudoaneurysm may have been triggered by ESWL.

To the best of our knowledge, this is the first case report in English of hemorrhagic pseudoaneurysm in a pancreatic pseudocyst after ESWL. We conclude that the risk of hemorrhagic pseudoaneurysm in a pancreatic pseudocyst must be considered during ESWL for pancreatolithiasis.
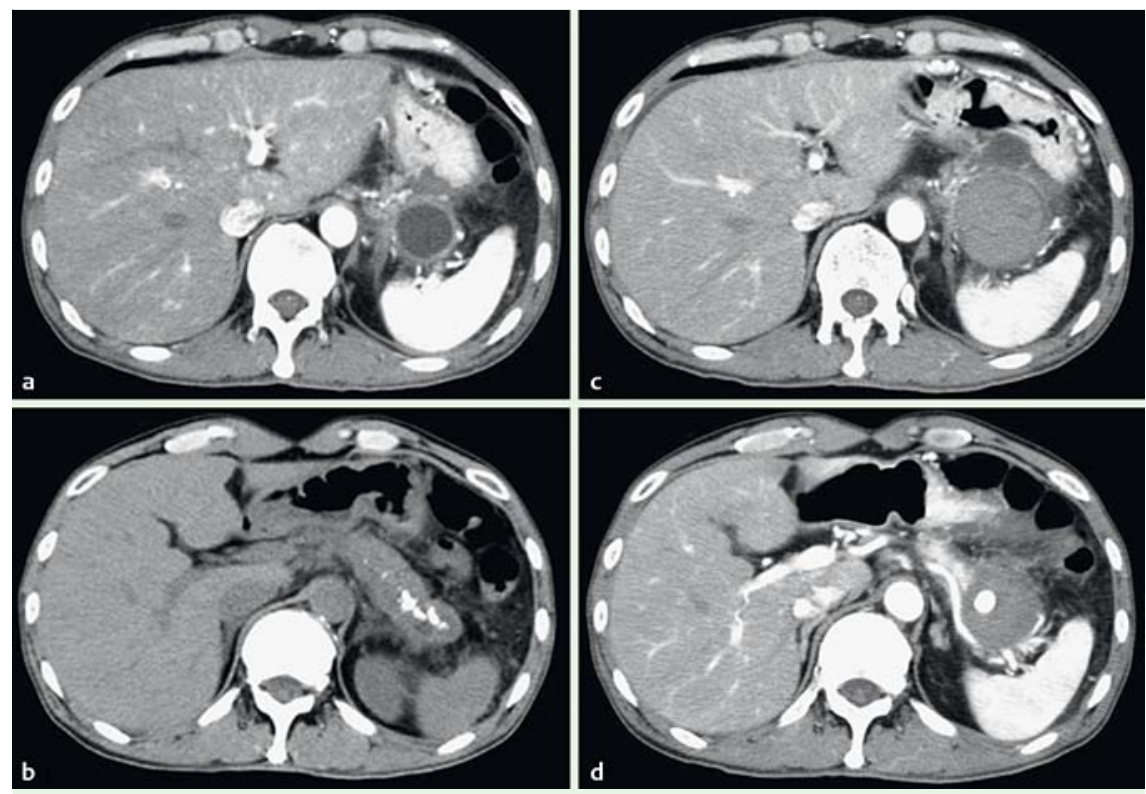

Fig. 1 Abdominal computed tomography (CT) scan showing: a a pseudocyst (30 mm in diameter) in the pancreatic tail, without a pseudoaneurysm, in a contrast-enhanced scan prior to extracorporeal shock wave lithotripsy (ESWL); b chronic pancreatitis with multiple calculi in an unenhanced scan prior to ESWL; c enlargement of the pseudocyst ( $50 \mathrm{~mm}$ in diameter) in a contrast-enhanced scan after ESWL; $\mathbf{d}$ a hemorrhagic pseudoaneurysm in the enlarged pseudocyst.

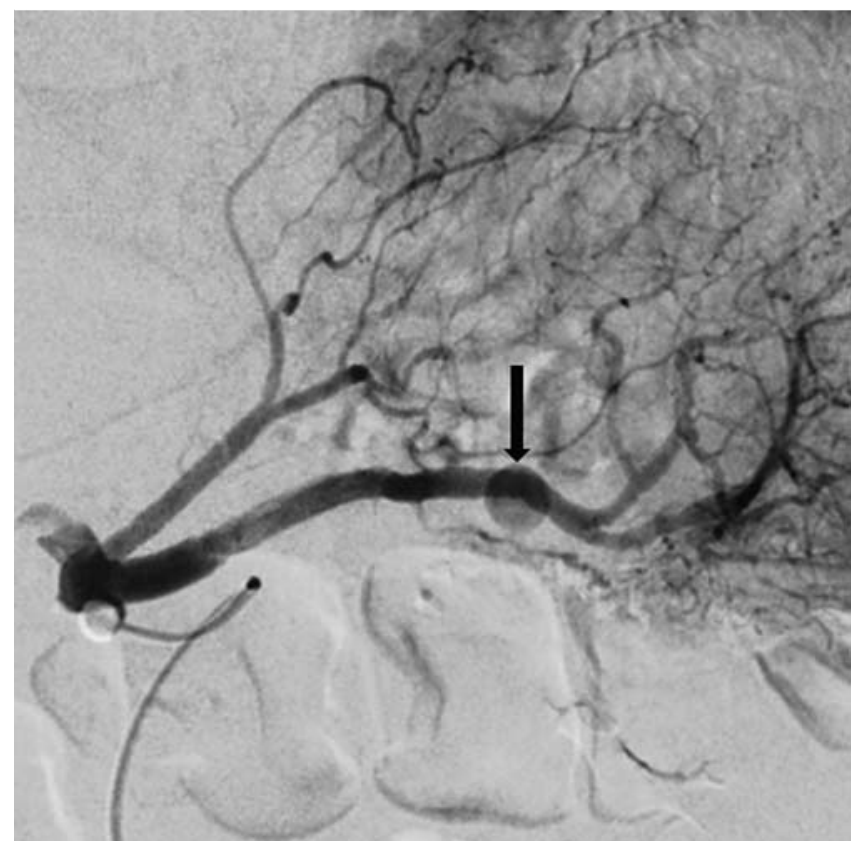

Fig. 2 Selective celiac angiography showing a pseudoaneurysm in the pancreatic branch of the splenic artery. 
Endoscopy_UCTN_Code_CCL_1AZ_2AH

Competing interests: None

Y. Nakagawa, T. Abe, M. Uchida,

K. Inoue, R. Ogawa, K. Mizukami, T. Okimoto, M. Kodama, K. Murakami, T. Fujioka

Department of General Medicine and Gastroenterology, Oita University, Yufu, Japan

\section{References}

1 Stabile BE, Wilson SE, Debas HE. Reduced mortality from bleeding pseudocysts and pseudoaneurysms caused by pancreatitis. Arch Surg 1983; 118: 45-51

2 Vujic I. Vascular complications of pancreatitis. Radiol Clin North Am 1989; 27: 81 - 91

3 Choi KS, Kim MH. Extracorporeal shock wave lithotripsy for the treatment of pancreatic duct stones. J Hepatobiliary Pancreat Surg 2006; 13: 86-93

4 Riedler I, Trummer H, Hebel P, Hubmer G. Outcome and safety of extracorporeal shock wave lithotripsy as first-line therapy of lower pole nephrolithiasis. Urol Int 2003; 71: $350-354$
Bibliography

DOI $10.1055 / \mathrm{s}-0030-1256642$

Endoscopy 2011; 43: E310 - E311

(c) Georg Thieme Verlag KG Stuttgart · New York . ISSN 0013-726X

\section{Corresponding author} Y. Nakagawa, MD

Department of General Medicine and Gastroenterology

Faculty of Medicine, Oita University

1-1 Idaigaoka Hasama-machi Yufu 879-5593 Japan

Fax: +81-97-5866194

nakagawa4423@ybb.ne.jp 